\title{
A machine-level software program for the generation of aversive conditioning schedules using the KIM-1 microprocessor
}

\author{
JAMES E. LAWLER and R. ALLAN BUCHHOLZ \\ University of Tennessee, Knoxville, Tennessee 37916
}

\begin{abstract}
A microprocessor system can be used both to control aversive conditioning (classical, signaled and Sidman avoidance, and conflict) experiments and to acquire behavioral data in multiple subjects at a fraction of the cost of solid state systems. The software described in this paper can be used to program tones and shocks and count responses, independently, in up to 12 animals simultaneously.
\end{abstract}

The development of inexpensive microprocessor (microP) systems allows the experimental psychologist to take advantage of the flexibility inherent in these systems in order to replace expensive and inflexible hard-wired solid state logic. The purpose of this paper is to explain the use of a machine-level software program for controlling the presentation of tones and shocks and recording responses with from 1 to 12 animals simultaneously, under various aversive conditioning paradigms.

The software was designed for use with the KIM-1 microP, which contains $1 \mathrm{~K}$ RAM, $2 \mathrm{~K}$ ROM, six-digit LED display, Teletype interface, 15-bit I/O port, audio cassette interface, hexidecimal keypad, and programmable clock. The hardware required for the software includes a Teletype and specialized interface developed in our laboratory (Murray \& Lawler, 1978). The complete system costs less than $\$ 325$, excluding the Teletype.

A block diagram of the hardware interface is depicted in Figure 1. The system is designed to process behavior responses from four sound-attenuated boxes, each containing three animals (Figure 2). Inputs from the 12 animals are processed by software recognition of distinctive bit patterns on the Port B word of the microP. Animal responses via microswitch are transduced through interface circuitry into clear 70-microsec TTL signals. Responses are visually displayed on an LED panel for the experimenter's convenience and are wire OR'ed to the IRQ pin of the microP, generating an interrupt for each response. The 8 bits of Port $\mathrm{A}$ on the microP control the independent presentation of

This work was supported by a grant-in-aid from the American Heart Association and by Research Grant HL-19680 from the National Heart, Lung, and Blood Institute, NIH. Address reprint requests to James E. Lawler, PhD, Department of Psychology, University of Tennessee, Knoxville, Tennessee 37916. Present address of R. Allan Buchholz: Department of Medical Psychology, University of Oregon Health Sciences Center, Portland, Oregon 97201. tones and shocks to each of the four boxes. Additional circuitry allows for individual control of tone volume and shock intensity.

The software may be used to generate classical aversive, signaled shock avoidance, Sidman avoidance, or conflict (avoidance-avoidance) paradigms. Additional

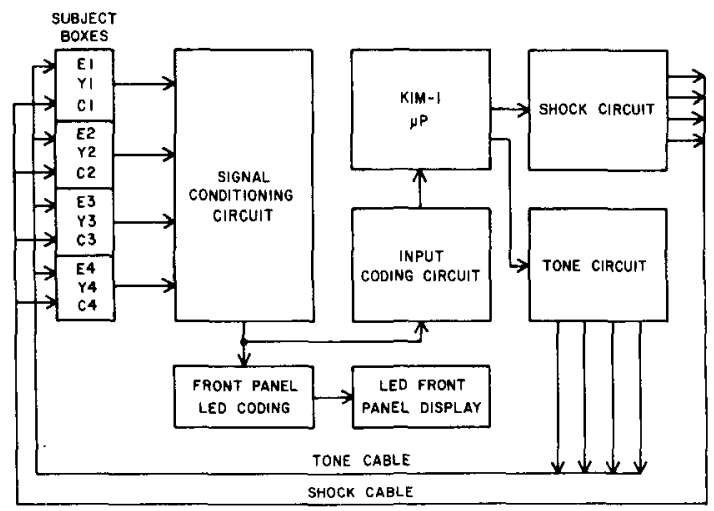

Figure 1. Block diagram of the microprocessor system (Murray \& Lawler, 1978).

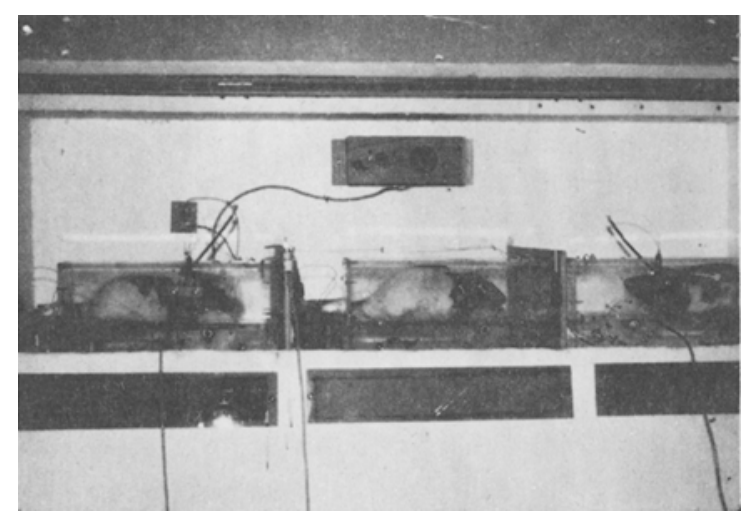

Figure 2. Interior of one sound-attenuated box containing (from left to right) one experimental, one yoked, and one nonshock control animal. 
features include the ability to run different boxes on different schedules, control of the S-S and R-S intervals, tone duration and shock duration (where relevant), precise timing of session duration with automatic session termination, and independent recording of shocks and tones presented to each box, as well as a count of responses made by each animal. A Teletype output routine displays the behavioral data.

Animals may be studied singly (one experimental subject), in pairs (including a yoked control), or in triplets (including a nonshock control). Up to four triplets may be run concurrently. The desired schedule(s) and appropriate timing intervals are defined in addresses 0001-0007 and are easily changed. These values must be hand entered before program execution. Addresses $0001-0004$ are used to program boxes on avoidance or conflict schedules. For classical conditioning, the contents of the four addresses are irrelevant. If used, the contents of the addresses must contain either OA (for avoidance) or OC (for conflict). Address 0001 is used to program Box 1 on avoidance or conflict; address 0002 is for Box 2, etc. Several avoidance schedules may be run concurrently if several animals are used. For example, if address 0001 contains OA, if 0002 contains OC, and if 0003 contains OA (with potentiometer off), then Subject 1 will be on signaled avoidance, Subject 2 on conflict, and Subject 3 on Sidman. In the classical conditioning mode, these addresses are irrelevant; however, the contents of address 00D9 must be changed from clear interrupt (58) to no operation (EA).

Timing intervals are stored in addresses 0005-0007. For time between last response (or shock) and tone onset, enter a hexidecimal code depicting the number of $.2 \mathrm{sec}$ intervals desired. For example, for $5 \mathrm{sec}$ enter 19 , for $10 \mathrm{sec}$ enter 32 , etc. This value is entered in address 0005 . Address 0006 contains the value representing tone duration (number of $.2-\mathrm{sec}$ intervals), which must be entered by hand using the same hexidecimal codes described above. Finally, the contents of address 0007 determine shock duration, which may vary from .2 to $2.0 \mathrm{sec}$. Again, the contents of the address specify duration in $.2-\mathrm{sec}$ intervals. Thus, 05 results in a $1-\mathrm{sec}$ shock. Shock can be programmed to pulsate by changing the contents of one address register (01C7). In our application, this register contains 06 , causing shock to pulsate once each second for $5 \mathrm{sec}$. For one shock, enter 02 in this address.

In the avoidance mode, a response by the experimental animal turns the tone off (if on) and resets timers to zero. In the conflict mode, timers are also reset by the experimental animal's response. However, in this mode, a response by the experimental animal before shock onset results in the delivery of one shock to both experimental and yoked subjects. Shock duration varies randomly from 0 to $200 \mathrm{msec}$. Failure to respond in the appropriate interval results in the delivery of multiple inescapable shocks (e.g., five), each $.2 \mathrm{sec}$ in duration (01 in address 0007).
The software consists of several routines. The initialization routine (1) clears appropriate registers used to count shocks, tones, and responses to zero; (2) sets up the interrupt vector address; (3) programs $\mathrm{I} / 0$ ports appropriately as input or output; (4) sets up the programmable clock to time the appropriate intervals. The timing routine examines counting registers for all experimental animals each $.2 \mathrm{sec}$ to determine if any animal is to receive a tone or shock. All intervals are independent for each experimental animal. The tone/ shock-Hi routine turns either tone or shock on to the appropriate experimental animal (and yoked subject, if used) when the correct interval has elapsed. The tone/shock-Lo routine turns either tone or shock off to the appropriate subject(s) when necessary. Finally, the interrupt service routine records responses from each animal independently in double-precision decimal registers that can thus count to 9999. Separate sets of registers count responses before, and after, shock onset. This routine also results in a JSR to tone/shock Lo if the experimental animal has responded at the appropriate time in the avoidance mode and a JSR to tone/ shock $H i$ if the experimental animal has responded at the appropriate time in the conflict mode.

Session duration is defined by the content of address 0082. This address contains the number of $1 / 2 \cdot h$ blocks desired. For example, for a $1 / 2-h$ session, enter 01 ; for a 2-h session, enter 04 , etc.

At the termination of the session, tones and shocks are discontinued, counting of responses ceases, and the LED display shows all zeros. The experimenter can then output the data to Teletype by starting the program at address 0300 . Data are output in the format depicted in Table 1. The terms B1, B2, etc., stand for Box 1, Box 2, etc., which can be composed simply of one experimental subject, or a pair (including $Y$ ), or triplet (including C). The word "TONE" refers to the number of tone presentations; "FAIL" refers to the number of failures to respond by experimental subjects during tone. Responses ("RESP") are output on six lines as follows: lines 1-3 contain responses before (" $B$ ") shock onset for $E, Y$, and $C$, respectively, for each box, while lines 4-6 contain responses after shock (" $A$ ") for the same groups.

The program has been in use for 1 year. Its major advantage is the minimal set-up time required. No

Table 1

Output Format for Behavioral Data

\begin{tabular}{lccccc}
\hline & & B1 & B2 & B3 & B4 \\
\hline FAIL & & 0000 & 0000 & 0000 & 0000 \\
TONE & & 0000 & 0000 & 0000 & 0000 \\
& E & 0000 & 0000 & 0000 & 0000 \\
RESP B & Y & 0000 & 0000 & 0000 & 0000 \\
& C & 0000 & 0000 & 0000 & 0000 \\
& E & 0000 & 0000 & 0000 & 0000 \\
RESP A & Y & 0000 & 0000 & 0000 & 0000 \\
& C & 0000 & 0000 & 0000 & 0000 \\
\hline
\end{tabular}


additional RAM is needed, other than that included on the KIM-1 board (1K words). In addition to the hardware interface described elsewhere (Murray \& Lawler, 1978), only four wires are needed to run a Teletype or serial DECwriter.

A listing of the program will be sent on request.

\section{REFERENCE}

Murray, D. M., \& LaWLer, J. E. A KIM-1 microprocessor interface for aversive conditioning applications using multiple subjects. Behavior Research Methods \& Instrumentation, 1978, 10, 334-339. 